\title{
Analysis of Sponsored Data Practices in the Case of Competing Wireless Service Providers
}

\author{
Patrick Maillé and Bruno Tuffin
}

\begin{abstract}
With wireless sponsored data, a third party, content or service provider, can pay for some of your data traffic so that it is not counted in your plan's monthly cap. This type of behavior is currently under scrutiny, with telecommunication regulators wondering if it could be applied to prevent competitors from entering the market, and what the impact on all telecommunication actors can be. To answer those questions, we design and analyze in this paper a model where a Content Provider (CP) can choose the proportion of data to sponsor and a level of advertisement to get a return on investment, with several Internet Service Providers (ISPs) in competition. We distinguish three scenarios: no sponsoring, the same sponsoring to all users, and a different sponsoring depending on the ISP you have subscribed to. This last possibility may particularly be considered an infringement of the network neutrality principle. We see that sponsoring can be beneficial to users and ISPs depending on the chosen advertisement level. We also discuss the impact of zero-rating where an ISP offers free data to a $\mathrm{CP}$ to attract more customers, and vertical integration where a CP and an ISP are the same company.
\end{abstract}

\section{Introduction}

With the improving capacity of smartphones, wireless data consumption is exponentially increasing. According to previsions ${ }^{1}$, mobile data consumption will be sevenfold larger by 2021 than by 2017. Wireless communication subscription offers are

Patrick Maillé

IMT Atlantique / IRISA, Rennes, France, e-mail: patrick.maille@imt.fr

Bruno Tuffin

Inria, Rennes, France e-mail: bruno.tuffin@inria.fr

1 see http://www.businessinsider.fr/us/mobile-data-will-skyrocket-700-by-2021-2017-2/ among others 
often made of unlimited telephony but caps on data over which a volume-based fee is applied. Users may therefore be forced to monitor and even limit their data usage.

Content providers (CPs) on the other hand are economically dependent on the amount of data consumed by users, typically via displayed advertisements: the more volume consumed, the more advertisement and then revenue. For this reason, there might be an incentive for content providers to sponsor data, that is, to at least partially pay the Internet Service Provider (ISP) for the user's consumption of its own services. This way, users may expand their usage, CPs may get more revenue due to advertisement, and ISPs are paid for the traffic increase. Examples in the USA are: Netflix or Binge-On with T-Mobile, DIRECTV and U-verse Data Free TV with AT\&T, etc. ISPs are even offering services such that CPs can, if they wish, apply sponsored data: examples are Verizon Wireless, AT\&T Mobility and T-Mobile US, or Orange with DataMI in France, among others.

But sponsored data bring concerns from user associations and small content providers. It is claimed to give an unfair advantage to some content/service providers and to eventually prevent some actors from entering the market, due to lower visibility and high entrance costs to the sponsored data system if they want to get the same service as incumbent and big providers already present. The culmination of the sponsored data principle is the so-called zero-rating where ISPs (freely) remove some content providers from their data caps, hoping more customers will subscribe due to potentially unlimited usage. Those providers could then hardly be challenged. The French ISP SFR did it with YouTube in its offer RED a few years ago. Similarly, Facebook, Google and Wikipedia have built special programs in developing countries, with the claimed goal to increase connectivity and Internet access. For those reasons, sponsored data and zero-rating are currently under investigation by regulators to determine if rules should be imposed to prevent or limit their use. In Chile for example, the national telecom regulator has stated that it was violating net neutrality laws and that it should be forbidden. Net neutrality means that all packets/flows are treated the same, independently of their origin, destination, and type of service $[3,4]$.

The purpose of this paper is to design and analyze a mathematical model of sponsored data to understand if this type of behavior should be banned or encouraged. To our knowledge, very few similar works exist, that we now summarize and from which we differentiate ourselves. The notable works are first [2], where the authors consider a model with a discrete set of users, a single ISP, and several (complement) CPs able to choose a different level of sponsoring per user. They show that sponsoring can benefit more to users than to CPs. Our model presents similarities with that one but strongly differs on many aspects: we use a different model for CPs' advertisement level and determination of the best strategy; our model also represents the negative externality of advertisements on users, something often forgotten, and a different model of volume consumption highlighting the correlation between willingness-to-pay for connectivity and consumed volume of data; finally and more importantly, we model competition between ISPs to better link the sponsored data practice to the network neutrality debate. We can also mention [7], dealing with several substitutable CPs in competition, or $[8,9]$ including network externalities, 
but again a single ISP, or [6] which nicely combines sponsored data and caching strategies, but not considering any ISP in the equation.

Our contributions are the following: i) the use of a model different from the literature to represent user behavior. This model separates the willingness-to-pay for connectivity and for CP content. Remark that using a new model is important to better understand the assumptions that may change the results, or see the robustness of conclusions. ii) We focus on CPs' strategical decisions and their impact on all other actors (ISPs and users). The decision variables are the amount (proportion) of data to be sponsored and the amount of advertisement CPs will set; our study allows us to see if sponsoring data also means more advertisement for a return on investment for CPs. iii) We investigate the best sponsoring and advertisement strategies in the case of competition between ISPs; three situations are compared: (a) no sponsored data (b) the same level of sponsoring at all ISPs (c) the possibility to sponsor differently according to the ISP; these situations also correspond to three different levels of neutrality in the net neutrality debate. iv) We investigate and compare with all other situations the case of zero-rating, when the CP content is not counted in the user data cap; ISPs could indeed be in favor of such a strategy if it brings additional subscriptions. v) Finally we look at the case when the $\mathrm{CP}$ is managed by one ISP, that is the so-called vertical integration; does it lead to an unfair advantage if data are sponsored with respect to a non-sponsored situation? This corresponds to practical situations within an increasingly vertically-integrated ecosystem.

The remainder of the paper is organized as follows. Section 2 presents the model, the decision variables and their hierarchy. Section 3 discusses the last level, how users distributes themselves among ISPs depending on prices and strategies of CPs. Then Section 4 describes the three sponsoring strategies and compares their impact on all actors in the case of independent CPs and ISPs. The particular situation of zero-rating, with the CP content not included in one ISP data plan but included in the other is investigated in Section 5. Section 6 presents the case of a verticallyintegrated CP-ISP. Finally, from all the described scenarios, we conclude in Section 7 on the need to regulate sponsored data.

\section{Model}

We consider three types of actors: users, CPs and ISPs. We describe here the model with full generality, and will later restrict the number of actors to focus on specificand to our knowledge un-investigated-aspects of the sponsored data debate.

Consider $M$ CPs indexed by $j$ and $N$ ISPs indexed by $i$. 


\subsection{Users}

We index users by $\theta \in \mathbb{R}^{+}$, which characterizes their type. We consider a continuum of users of total mass 1 , and denote by $F$ the cumulative distribution function of $\theta$. Each user $\theta$ has several variables of decision: its choice of ISP, that we will denote by $i(\theta)$, but also the volume of $\mathrm{CP} j$ data (without advertisements) he will consume if using ISP $i$, which we will denote by $v_{i, j}(\theta)$. The dependence on $i$ comes from the fact that prices for data may differ between ISPs, and the consumed volume too as a consequence.

To simplify, let $\theta$ be the willingness-to-pay for connectivity and $p_{i}$ the subscription price at ISP $i$, which would be giving a "utility for connectivity" $\theta-p_{i}$. But we additionally weigh the difference using an ISP reputation parameter $a_{i} \geq 0$, meaning that users value more the gain obtained with incumbent providers than with newcomers, resulting in a "weighted utility for connectivity"

$$
a_{i}\left(\theta-p_{i}\right)
$$

In addition, User $\theta$ gains satisfaction from using each $\mathrm{CP} j$. The cost per unit of volume he pays in his data plan is denoted by $c_{i, j}$, which depends on $j$ because that usage can be sponsored by $\mathrm{CP} j$ as we will see in the next subsection. Moreover there is a valuation for each unit of volume of CP $j$. Let $r_{\theta, j}^{\prime}$ be the marginal valuation, that we will consider to be

$$
r_{\theta, j}^{\prime}(x)=\left[\theta-\left(\alpha_{j} s_{j}^{2}\right) x\right]^{+}
$$

for the $x$-th unit of useful volume, i.e., without advertising, where $[y]^{+}:=\max (y, 0)$, $\alpha_{j}$ is a fixed parameter, and $s_{j} \geq 1$ corresponds to the relative increase of volume due to advertisement displayed by $\mathrm{CP} j$, expressed as the total downloaded volume divided by the volume of "useful" data, excluding ads. The larger this value of $s_{j}$, the smaller the service valuation for users. We consider a square value of $s_{j}$ to assure the reasonable assumption that at one point, for the CPs, the loss due to users unpleased by advertisements exceeds the gain from those ads (see later (2) and (3) with $s_{j}$ tending to infinity); any other choice can be used without difficulty though. Then the willingness-to-pay $r_{\theta, j}(x)$ for consuming a volume $x$ of $\mathrm{CP} j$ data over a month is

$$
r_{\theta, j}(x)= \begin{cases}\theta x-\alpha_{j} s_{j}^{2} \frac{x^{2}}{2} & \text { if } x \leq \frac{\theta}{\alpha_{j} s_{j}^{2}} \\ \frac{\theta^{2}}{2 \alpha_{j} s_{j}^{2}} & \text { otherwise. }\end{cases}
$$

This form is commonly adopted in $[1,5]$ and presents the advantage of positively correlating willingness-to-pay for data and willingness-to-pay for connectivity. We have added in the model the negative correlation due to advertisement (some kind of "pollution" which can advert users). Note that a positive effect (only) from advertisement was considered in [2] due to relevant ads which can be clicked, but ads seems to us rather negatively perceived.

Our assumptions give a utility for user $\theta$ at ISP $i$ 


$$
U_{i}(\theta):=a_{i}\left(\theta-p_{i}\right)+\sum_{j=1}^{M}\left(r_{\theta, j}\left(v_{i, j}(\theta)\right)-c_{i, j} v_{i, j}(\theta) s_{j}\right),
$$

using the fact that the total downloaded volume is actually $v_{i, j}(\theta) s_{j}$ from the definition of $s_{j}$. Users indeed also download advertisements, which are not differentiated from "real" content by ISPs. The additive expression indicates that CPs are assumed to be independent in terms of content.

Our modeling, unlike those of the literature ([2] for example), chooses to represent user preferences for ISPs, in terms of connectivity and a correlation between willingness-to-pay for connectivity and consumed volume of data; it separates what comes from ISPs from what comes from CPs.

If subscribing to ISP $i$, the volume $v_{i, j}(\theta)$ User $\theta$ chooses is the one maximizing $r_{\theta, j}\left(v_{i, j}(\theta)\right)-c_{i, j} s_{j} v_{i, j}(\theta)$, and can easily be computed [1] to be

$$
v_{i, j}(\theta)=\left[\frac{\theta-c_{i, j} s_{j}}{\alpha_{j} s_{j}^{2}}\right]^{+},
$$

leading to $r_{\theta, j}\left(v_{i, j}(\theta)\right)-c_{i, j} s_{j} v_{i, j}(\theta)=\frac{\left(\theta-c_{i, j} s_{j}\right)^{2}}{2\left(\alpha_{j} s_{j}^{2}\right)} \mathbb{1}_{\left\{\theta>c_{i, j} s_{j}\right\}}$.

Summarizing, User $\theta$ chooses ISP $i(\theta)$ maximizing his overall utility:

$$
i(\theta)=\operatorname{argmax}_{i} U_{i}(\theta)=\operatorname{argmax}_{i} a_{i}\left(\theta-p_{i}\right)+\sum_{j=1}^{M} \frac{\left(\theta-c_{i, j} s_{j}\right)^{2}}{2\left(\alpha_{j} s_{j}^{2}\right)} \mathbb{1}_{\left\{\theta>c_{i, j} s_{j}\right\}}
$$

if the max is non-negative, otherwise $i(\theta)=0$, meaning no subscription at all.

\section{$2.2 C P S$}

$\mathrm{CP} j$ is assumed to have a utility (a revenue) linearly increasing with the volume of displayed advertisement, with CP-dependent linear parameter $\beta_{j}$. The advertisement volume is the total volume $s_{j} v_{i(\theta), j}(\theta)$ minus the "real" data volume $v_{i(\theta), j}(\theta)$, hence $\left(s_{j}-1\right) v_{i(\theta), j}(\theta)$. As a consequence, the gain for $\mathrm{CP} j$ is

$$
\int_{\theta} \beta_{j}\left(s_{j}-1\right) v_{i(\theta), j}(\theta) \mathrm{d} F(\theta) .
$$

Each CP $j$ can decide to sponsor a fraction $\gamma_{i, j}$ of the data usage cost of ISP $i$ 's users. This could be an incentive to consume more $\mathrm{CP} j$ content and therefore generate more revenue from advertisement. Of course, there is a trade-off, which is one purpose of this paper, between sponsoring cost and generated volume of consumed data. We will investigate three different sponsoring policies:

1. No sponsored data: $\gamma_{i, j}=0 \forall i, j$;

2. The same data sponsoring level for all ISPs: $\gamma_{i, j}=\gamma_{j} \forall i, j$; 
3. A possible differentiation between ISPs, with $\gamma_{i, j} \neq \gamma_{i^{\prime}, j}$ for $i \neq i^{\prime}$.

In terms of the network neutrality debate, those three scenarios correspond to three different levels of neutrality. Option 1 corresponds to full neutrality. Option 2 seems neutral with respect to ISPs, even if non-neutral between applications because different applications can have different levels of sponsoring (note on the other hand that advocates of sponsored data also claim that packets are treated the same within the network, but it is not the issue we want to address here). Option 3 is the fully non-neutral one.

Let $q_{i}$ be the unit price ISP $i$ is (officially) charging users for data. After data sponsoring, the unit cost seen by users when consuming data of $\mathrm{CP} j$ is then

$$
c_{i, j}=q_{i}\left(1-\gamma_{i, j}\right) .
$$

If sponsoring data, $\mathrm{CP} j$ has to pay to each ISP for the proportion of volume it has chosen to sponsor, hence a total cost: $\int_{\theta} \gamma_{i(\theta), j} q_{i(\theta)} s_{j} v_{i(\theta), j}(\theta) \mathrm{d} F(\theta)$, where the proportion of volume paid, is the proportion $\gamma_{j}$ of the total volume $s_{j} v_{i(\theta), j}(\theta)$, (which includes ads).

Compiling all the elements, the revenue of $\mathrm{CP} j$ is

$$
G_{j}=\int_{\theta}\left(\beta_{j}\left(s_{j}-1\right)-\gamma_{j} q_{i(\theta)} s_{j}\right) v_{i(\theta), j}(\theta) \mathrm{d} F(\theta) .
$$

$\mathrm{CP} j$ has several decision variables: the sponsoring levels $\gamma_{i, j} \forall i$ and the advertisement volume increase level $s_{j} \geq 1$. Note that we could also consider advertising levels that would depend on the user ISP (i.e., $s_{i, j}$ instead of $s_{j}$ ): we rather impose an equal advertising level, since our focus is on the sponsoring strategies, and CPs may not know the ISP of the user upon a request for content.

\subsection{ISPS}

Each ISP $i$ tries to maximize its revenue. Revenue comes from subscriptions and consumed data:

$$
R_{i}=\int_{\theta}\left(p_{i}+\sum_{j} q_{i} s_{j} v_{i, j}(\theta)\right) \mathbb{1}_{\{i(\theta)=i\}} \mathrm{d} F(\theta) .
$$

\subsection{What we analyze}

Our purpose in this paper is to analyze the impact of the decisions of CPs on all actors, in the context of competing ISPs. In particular, we would like to see the impact of a "neutrally" sponsoring CP (that is, $\gamma_{i, j}=\gamma_{i^{\prime}, j}$ for any ISPs $i, i^{\prime}$ ) with respect to sponsoring differently depending on ISPs and with respect to no sponsoring. 
Three scenarios are analyzed in the next sections:

1. In the case of independent ISPs and CPs, what are the best advertising and sponsoring levels of CPs and the impact on all actors? We can compare the outputs with optimal solutions for the three sponsoring possibilities.

2. Does zero-rating, where an ISP leaves a CP out of its data plan to try to attract customers, harm users and the other ISPs? Should it be forbidden?

3. Does vertical integration of a CP and an ISP have a positive or negative impact on the other ISPs and on users? This, with a dominant or a non-dominant ISP.

Since CPs are complements (due to the additive form of users' utility), we will consider without much loss of generality in the next sections the case of a single CP. This in order to focus on our purpose, that is the impact of advertisement and sponsoring strategies on competition between ISPs, and on users. Hence we can simplify the writing by removing the CP-relative indices in the notations defined before. We will also limit ourselves to 2 ISPs, labeled 1 and 2 .

\section{User subscription decisions}

User decisions depend on prices set by ISPs, but also on decisions of the CP. Even if the CP "plays" first, its optimal strategy should anticipate users' choices. For this reason, we discuss here the repartition of users depending on fixed $\mathrm{CP}$ decisions. The optimal CP decisions are analyzed in the next sections, anticipating this subsequent choice (this implicitly assumes that the $\mathrm{CP}$ knows the distribution $F$ ).

In other works using the same type of models for user preferences [1, 5], we had depending on the value of $\theta$ an interval over which users do not subscribe, then an interval (possibly empty) over which one ISP is chosen, and then another one for the other ISP (that is, users with small valuations do no subscribe, those with "intermediate" valuation go with one ISP and those with the largest go with the other). Here things can be more complicated, with interestingly more intricate intervals. To illustrate this, consider the values $a_{1}=1.3, a_{2}=1, s=1, \alpha=1$, $c_{1}=2, c_{2}=1, p_{1}=1$, and $p_{2}=0.97$, i.e., ISP 1 has a better reputation but higher subscription and usage prices. Figure 1 displays the difference $\max \left(U_{1}(\theta), 0\right)-$ $\max \left(U_{2}(\theta), 0\right)$ in terms of $\theta$. We consider the max with 0 to show only the situations where a user $\theta$ is willing to subscribe to an ISP.

We can see (when the curve gives zero) that users with small valuations $\theta$ prefer not to subscribe to any ISP; then there is an interval where ISP 2 is preferred, then an interval where it is ISP 1, and then again ISP 2. An interpretation is as follows: users with small valuation (but large enough to subscribe) are first interested in connectivity with a smaller connectivity price at ISP 2 . At one point, due to its larger reputation, connectivity attractiveness of ISP 1 becomes larger, but it is counterbalanced by the data cost of ISP 2 (the parabola).

In general, in terms of the combination of prices, we can express how many such successive intervals above the "no subscription" possibility can be found, between 


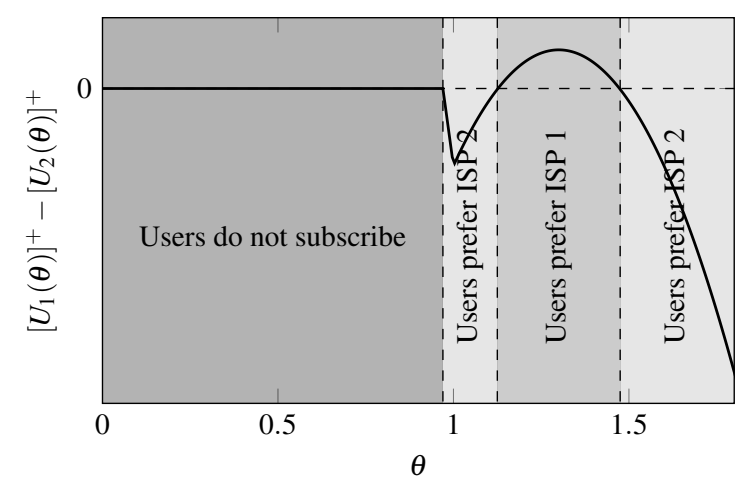

Fig. $1\left[U_{1}(\theta)\right]^{+}-\left[U_{2}(\theta)\right]^{+}$in terms of $\theta$. In the left zone (small $\theta$ values), users do not subscribe to any provider.

one and three. We do not give such details here since it is a list of cases which is not very instructive. The situation becomes even more intricate if we increase the number of ISPs (and CPs). Solving it numerically in the next section does not pose any problem.

\section{Analysis with independent CP and ISPs}

In all our numerical investigations, $\theta$ follows an exponential distribution with rate 1 truncated at 2, i.e., $F(\theta)=\left(1-e^{-\theta}\right) /\left(1-e^{-2}\right)$ for $\theta \in[0,2]$. Unless specified otherwise (i.e., when a parameter explicitly varies), the parameter values we consider throughout all the paper are summarized in Table 1. ISP 1 has the position

$$
\begin{array}{llllllll}
a_{1} & a_{2} & p_{1} & p_{2} & q_{1} & q_{2} & \beta & \alpha \\
\hline 1.5 & 1 & 1 & 0.6 & 0.6 & 0.4 & 1 & 1
\end{array}
$$

Table 1 Parameter values for the numerical investigations

of an incumbent: with a better reputation than its competitor, it can charge higher subscription and per-usage prices.

We discuss in this section the case where the ISP and CP are independent. We display in Figure 2 the CP revenue as $s$ varies for fixed values of the other parameters, including fixed values of the sponsoring coefficient $\gamma_{i}$ on ISP $i=1,2$, but using the subsequent user subscription decision. It can be checked that there is an optimal level of advertising $s^{*}$ around 2.7 above which increasing the displayed ads diminishes revenue.

In Figure 3 we display the CP revenue-maximizing sponsoring levels $\gamma_{i}$ as $s$ varies, and the corresponding CP Revenue. We can see that the optimal $\gamma_{i}$ is in- 


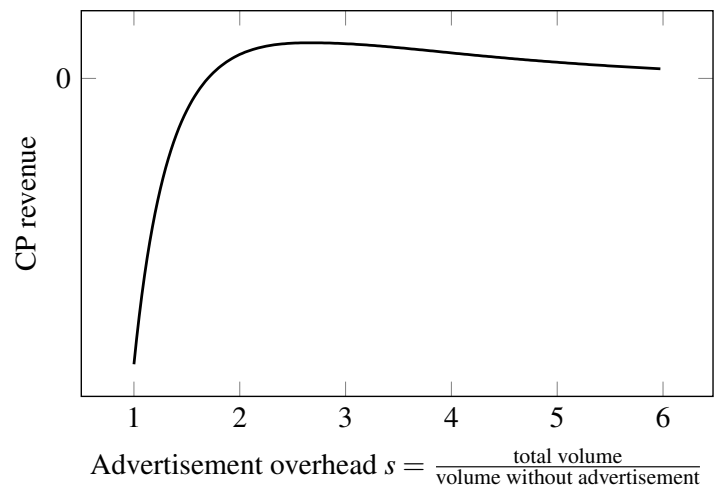

Fig. 2 CP revenue, with $\gamma_{1}=0.7, \gamma_{2}=0.4$.
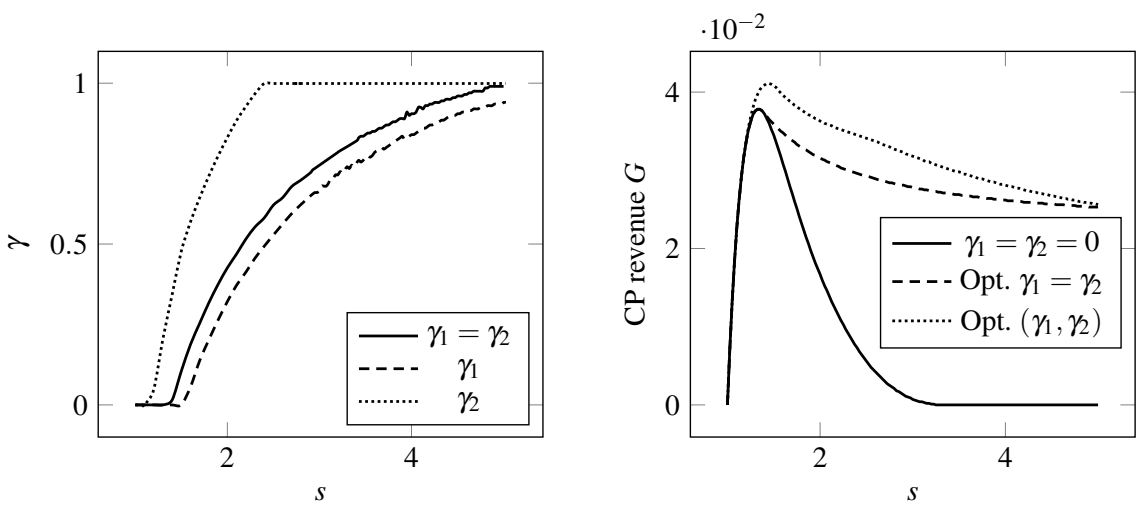

Fig. $3 \mathrm{CP}$ revenue-maximizing sponsoring coefficient $\gamma$ (left), and corresponding $\mathrm{CP}$ revenue (right) in each scenario.

creasing with the level of advertisement $s$ : the more ads you put, the more you can sponsor data because you earn more, and the more you have to do to compensate the negative externality for users. Typically also, applying the same level of sponsoring $\left(\gamma_{1}=\gamma_{2}\right)$ leads to a sponsoring level in between the differentiated levels of sponsoring $\left(\gamma_{1} \neq \gamma_{2}\right)$. Similarly to Figure 2 where sponsoring was fixed, in the present case of optimal sponsoring for each $s$, there is an optimal advertising level for each sponsoring scenario in Figure 3 (right). As expected, the CP revenue with differentiated sponsoring is larger than the one with identical sponsoring, itself larger than the one with no sponsoring, because the optimization is on a larger set of sponsoring parameters. When the advertisement level is low, there is no significative difference between the three options; it is significant when $s$ is large. The optimal advertising levels $s^{*}$ are 1.34 with no sponsoring, 1.34 too with identical sponsoring, and 1.44 with differentiated sponsoring, a larger value. "No sponsoring" or "identical sponsoring" yield a similar optimal revenue, while it is significantly better for differentiated sponsoring which then seems the appropriate option for the $\mathrm{CP}$. 

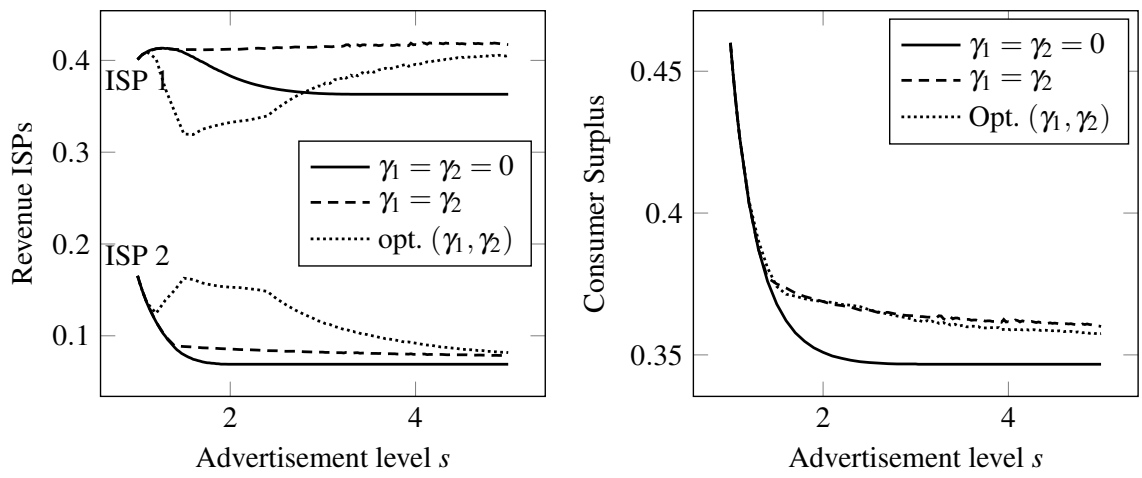

Fig. 4 ISP revenues (left) and Consumer Surplus (right) with CP-revenue-maximizing $\gamma$ values.

Figure 4 displays the impact of sponsoring on the revenues of ISPs and on consumer surplus. In terms of ISP revenue first, we can see that ISP 1 , the one with the highest reputation, makes more money than ISP 2. For very low values of $s$ the three policies give the same revenues. ISP 2 always prefers sponsoring to no sponsoring, while for ISP 1 an identical sponsoring is always the best option, something not so obvious at first sight. As a second choice for ISP 1, depending on the advertisement level, preference varies between no sponsoring and a differentiated one. Sponsoring is also always preferred by users, since giving a higher consumer surplus. On the other hand, the consumer surplus decreases as the advertisement level increases: the increased sponsoring level does not compensate enough the negative externality of ads. Interestingly, there is no significant difference for users between the two sponsoring options, and no dominance either.

From the figures, sponsoring seems a relevant option for all actors, with some differences in preferences between the two sponsoring options depending on the actors.

Table 2 compares the outputs at the optimal advertising levels selected by the CP. Note that in the case of identical sponsoring policy, the optimal advertising level is

\begin{tabular}{lcccccc} 
& $s^{*}$ & $\gamma_{i}$ & $G$ & $R_{1}$ & $R_{2}$ & $C S$ \\
\hline No sponsoring & 1.34 & $(0,0)$ & 0.0378 & 0.4124 & 0.0931 & 0.3813 \\
\hline Id. sponsoring & 1.34 & $(0,0)$ & 0.0378 & 0.4124 & 0.0931 & 0.3813 \\
\hline Dif. sponsoring & 1.45 & $(0,0.41)$ & 0.0411 & 0.3317 & 0.1559 & 0.3770
\end{tabular}

Table 2 Comparison of the three sponsoring scenarios with CP-revenue-maximizing advertisement and sponsoring levels, for the parameter values of Table 1 .

such that there is no sponsoring at all. Hence no sponsoring and identical sponsoring lead to the same output with our set of parameters. Differentiated sponsoring leads to slightly more ads. With this optimal choice, only customers of ISP 2 get sponsored data, $41 \%$ of data being sponsored. It leads to a larger CP revenue than the two other options. The revenue of ISP 1 decreases because attracting less customers, 
to the benefit of ISP 2. Consumer surplus is then slightly decreased. In conclusion, with this set of parameters and optimal advertising, identical sponsoring is of no help since leading to no sponsoring at all, and differentiated sponsoring just slightly changes consumer surplus but really modifies ISP revenues to the advantage of the one with the lowest reputation; this could be beneficial for competition with incumbent providers having usually a better reputation than newcomers.

\section{Zero-rating}

Zero-rating consists in leaving a $\mathrm{CP}$ out of the data plan, making it free of access for users. The potential interest for an ISP is to attract more customers, and therefore compensate the volume-based revenue loss by a subscription revenue increase. We study here whether this kind of strategy makes sense and what is the impact on all actors. Implementing zero-rating translates into $c_{i, j}=0$. In the case of a single CP, it is equivalent to considering $q_{i}=0$.

Figures 5 to 8 alternatively display the outputs in the cases where ISP 1 or ISP 2 implement zero-rating. Looking at the two scenarios helps to see if zero-rating is more relevant and has more impact depending on the market position of the ISP. Indeed, by keeping all values of parameters the same as in the previous section, ISP 1 is more established than ISP 2.

We see in Figure 5 (left) that in the case of zero-rating for ISP 1, the optimal sponsoring strategy for ISP 2 does not impact much the CP revenue, whatever the level of advertising. With respect to no zero-rating in Figure 3, the CP gain is substantial. Similar results can be observed when it is ISP 2 which applies zero-rating:
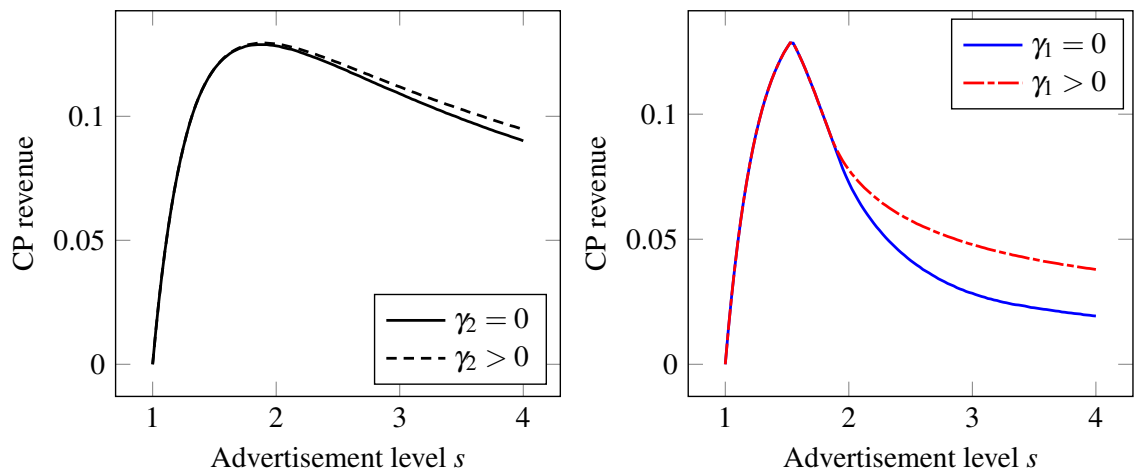

Fig. 5 CP revenue with zero-rating for ISP 1 (left) or for ISP 2 (right).

substantial gain for the CP, but at a smaller advertising level than when it is applied by ISP 1, while at high advertising levels the difference between no sponsoring or sponsoring for ISP 1 is significant. At the optimal advertising level, there is no dif- 
ference though. In Figure 6, we can see that, again, the sponsoring level increases

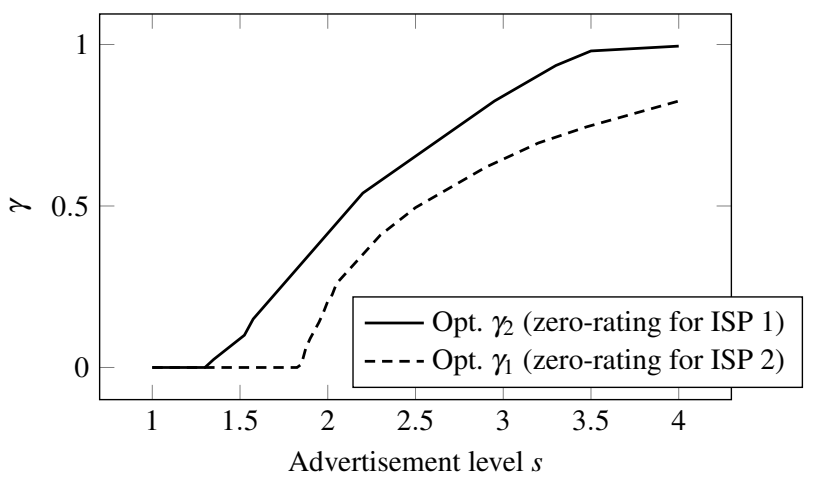

Fig. 6 Optimal $\gamma$ for an ISP, with zero-rating for the other ISP.

with the level of advertisement. But it can be checked that for smaller advertising levels, there is no sponsoring (which is understandable due to the low(er) revenue of the CP based on visits) and that sponsoring (for ISP 2 users) starts at a smaller advertising level for zero-rating with ISP 1 than in the opposite situation.
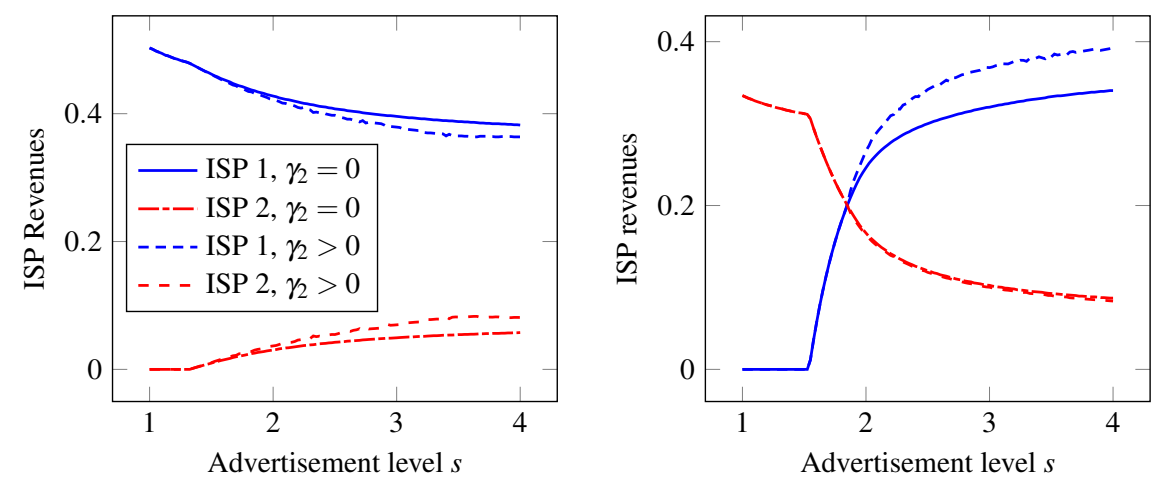

Fig. 7 ISP revenues with zero-rating for ISP 1 (left) and for ISP 2 (right).

In terms of ISP revenue in Figure 7, a zero-rating at ISP $i$ induces for small advertising levels no revenue at the other ISP, which can be seen as against competition and could lead to action from a regulator, especially given that the optimal advertising levels are actually quite low from Figures 5 to 8 . Sponsoring tends to reduce the gap in revenue with respect to no sponsoring when zero-rating is applied at ISP 1 only, while it is the opposite when applied at ISP 2.

Interestingly in Figure 8, consumer surplus does not change much with the policy if zero-rating is applied at ISP 1, the largest ISP. It changes a bit more when it is 


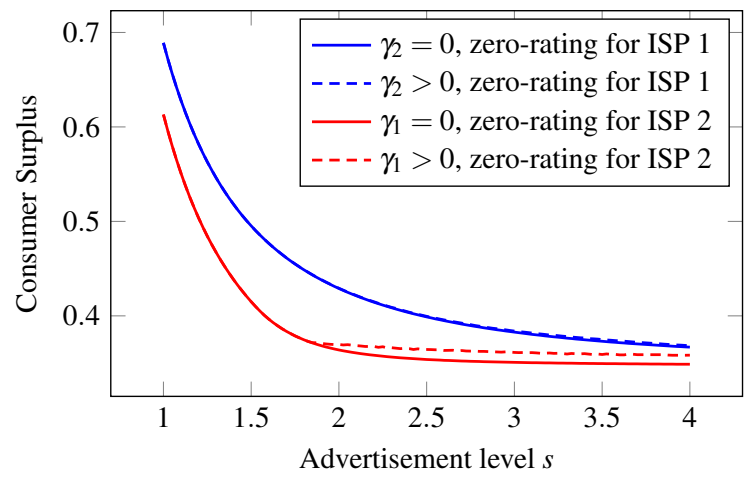

Fig. 8 Consumer Surplus with zero-rating for one ISP.

applied at ISP 2, with large levels of advertising only. In both cases anyway, note that sponsoring is beneficial to users, but at the advertising level chosen optimally by the $\mathrm{CP}$, there is no real difference (because the selected value is $\gamma_{1}=0$ in case of zero-rating at ISP 2).

\section{Vertical Integration}

We know look at the case of vertical integration: when the CP and one of the ISPs are the same firm. This situation becomes increasingly frequent in practice, with ISPs offering video-on-demand services, among other. In that case the optimization of parameters (sponsoring and advertising) is based on the combined revenues of the $\mathrm{CP}$ and the ISP, by just adding them.

Figure 9 (left) displays the revenue of the integrated CP-ISP in terms of $s$, in the cases where it is either ISP 1 or ISP 2 which is integrated, and with fixed sponsoring levels. ISP 1 being more established, it is not surprising that integrating it yields a larger revenue. But the optimal advertising level is larger in the case of ISP 2 with those parameters. Figure 9 (right) shows the revenue of the integrated entity (CPISP 1, or CP-ISP 2) with the corresponding optimal $\gamma$. Recall that in the case of non-integration, the optimal advertisement level was 1.35 for no or identical sponsoring, and 1.45 for differentiated sponsoring. We still have the dominance such that differentiated sponsoring is better than identical one, itself better than no sponsoring. It can be seen that for an integrated CP-ISP 1, identical sponsoring leads to the most ads (a level around 1.6, and 1.0-i.e., no ads-for no sponsoring and 1.4 for a differentiated one), while it is differentiated sponsoring in the case of an integrated CP-ISP 2 (a level around 1.65, and 1.05 both for no sponsoring and for an identical one). So the optimal advertising level maybe less or more for the same sponsoring policy if the $\mathrm{CP}$ is integrated or not. But if choosing the optimal policy, advertising is larger if vertical integration is applied. 

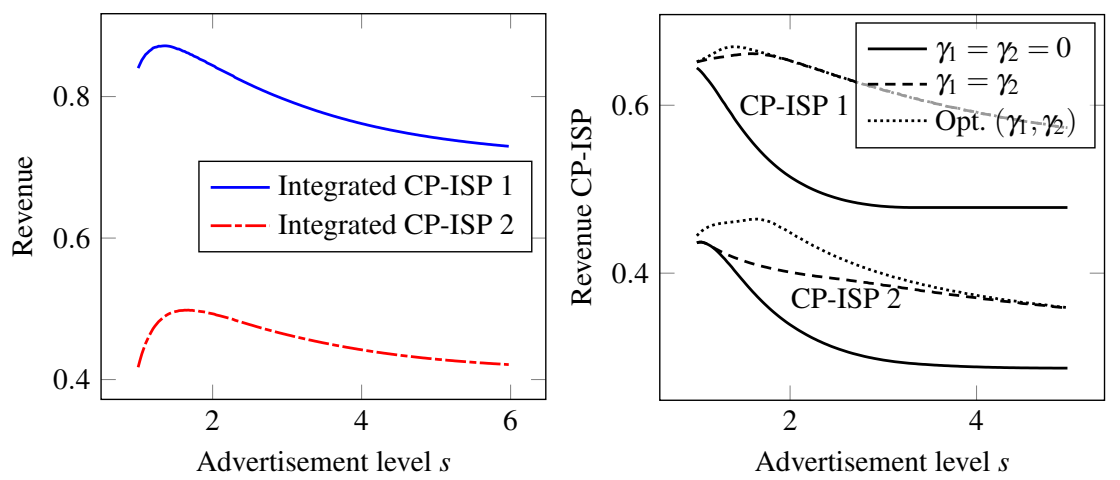

Fig. $9 \mathrm{CP}+\mathrm{ISP}$ revenue in the two integration scenarios, when $\gamma_{1}=0.7, \gamma_{2}=0.4$ (left), and with optimized values of $\left(\gamma_{1}, \gamma_{2}\right)$ (right).
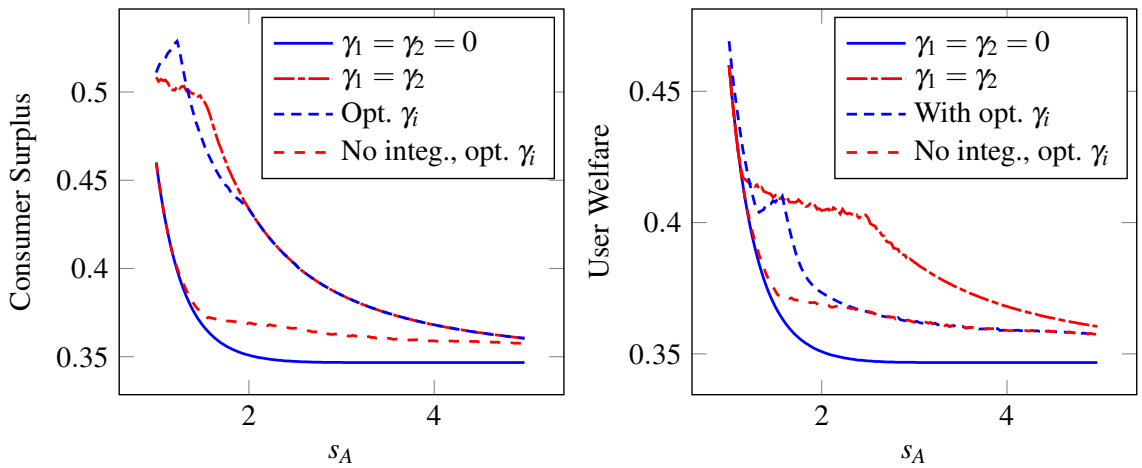

Fig. 10 Consumer surplus with integrated CP-ISP 1 (left) or integrated CP-ISP 2 (right), for $\beta_{A}=$ $1.00, \beta_{B}=1.50, r_{1}=1.50, r_{2}=1.00, p_{1}=1.00, p_{2}=0.60, q_{1}=0.60, q_{2}=0.40, \alpha_{A}=1.00, \alpha_{B}=$ $1.00, s_{B}=5.00$.

Figure 10 gives the consumer surplus in terms of $s$ for the two integration cases, and compares it with the optimal non-integrated strategy obtained in Section 4. It allows to see if integration has a positive or negative impact on users, something of interest for the regulator. We observe that vertical integration is good for users, especially if sponsoring data is allowed. An identical sponsoring is the best option with this regard. The reason is that it induces much more data sponsoring resulting in lower user cost.

Finally, Figure 11 displays the revenue of the non-integrated ISP for all strategies, in order to illustrate whether integration brings down competition. We also again plot the revenues in cases of no integration for comparison sake. An integrated ISP 1 reduces drastically ISP 2 revenue, which was expected, but at a lesser extent for the identical sponsoring strategy because of less freedom. Integrating the incumbent ISP is typically against competition. Surprisingly, when it is ISP 2 who is integrated and 

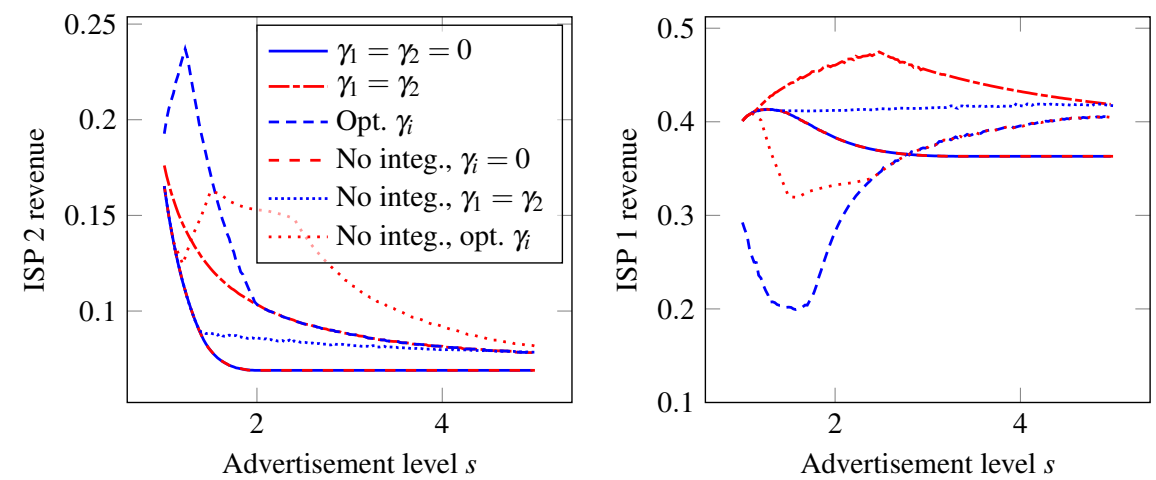

Fig. 11 Revenue of the non-integrated ISP if ISP 1 (left) or ISP 2 (right) is integrated.

with identical sponsoring, ISP 1 can benefit from the integration of the competitor. Note it is not the case for the other sponsoring strategies.

Table 3 compares the outputs at the optimal advertising levels selected by the $\mathrm{CP}$ in the two integrated scenarios. They can be compared with the non-integrated case of Table 2. Vertical integration, except partially for the last line of Table 3,

\begin{tabular}{|c|c|c|c|c|c|c|c|c|}
\hline & \multicolumn{4}{|c|}{$\mathrm{CP}$ integrated with ISP 1} & \multicolumn{4}{|c|}{$\mathrm{CP}$ integrated with ISP 2} \\
\hline & $s^{*}$ & $\left(\gamma_{1}, \gamma_{2}\right)$ & $G+R_{1}$ & $\mathrm{CS}$ & $s^{*}$ & $\left(\gamma_{1}, \gamma_{2}\right)$ & $G+R_{2}$ & $\mathrm{CS}$ \\
\hline No spons. & 1.0 & $(0,0)$ & 0.6445 & 0.16530 .4600 & 1.05 & $(0,0)$ & 0.4370 & $\overline{0.40620 .4420}$ \\
\hline Id. spons. & 1.6 & $.998,0.998)$ & 0.6614 & 0.11710 .4833 & 1.05 & $(0,0)$ & 0.4370 & 0.40620 .4420 \\
\hline Dif. spons. & 1.4 & $.783,0.995)$ & 0.6697 & 0.19250 .4882 & 1.65 & $243,0.997)$ & 0.4644 & 20210.3978 \\
\hline
\end{tabular}

Table 3 Output at optimal $s$ when the CP is integrated with an ISP.

always significantly benefits to users and competitors, something not so intuitive. We see in Table 3 that vertical integration leads to more sponsoring than in Table 2, so that consumer surplus is increased. With an integrated CP-ISP 2, the increased satisfaction in the first two lines is due to the smaller optimal advertising level; for the last line, the advertising level is larger but only partially counter-balanced by the increased sponsoring.

\section{Conclusions}

The purpose of this paper was to study the impact of sponsored data, differentiated or not, on all telecommunication actors, in the presence of competing ISPs. Our main results are the following:

- Sponsoring can be beneficial to users and ISPs and the impact depends on the chosen advertisement level. 
- Zero-rating at one ISP can lead to no revenue at the other ISP, which can be seen as harming competition.

- Vertical integration can be beneficial for all actors in this context, if the CP is integrated to the incumbent ISP (the one with the largest reputation).

Conclusions of course depend on the arbitrarily chosen set of parameters, but the paper provides insight on the potential effects of sponsored data, and a model to perform such an analysis.

We plan to extend the promising first results of this work in one main direction: We aim at inserting another round of decisions, on ISP prices (for subscription and for data usage). Our purpose here was to see the impact of having several ISPs on the $\mathrm{CP}$ decisions leading to important conclusions. Inserting a pricing game will complete the analysis but is computationally demanding. Other interesting extensions include considering a larger set of ISPs to show that the obtained results are without loss of generality in this respect, as well as considering CPs which can be substitutes.

\section{References}

1. M. Cho and M. Choi. Pricing for mobile data services considering service evolution and change of user heterogeneity. IEICE Transactions on Communications, E96-B(2):543-552, 2013.

2. C. Joe-Wong, S. Ha, and M. Chiang. Sponsoring mobile data: An economic analysis of the impact on users and content providers. In Proc. of INFOCOM 2015, Hong-Kong, China, 2015.

3. P. Maillé, P. Reichl, and B. Tuffin. Internet governance and economics of network neutrality. In A. Hadjiantonis and B. Stiller, editors, Telecommunications Economics - Selected Results of the COST Action IS0605 EconTel, pages 108-116. Lecture Notes in Computer Science 7216, Springer Verlag, 2012.

4. P. Maillé, G. Simon, and B. Tuffin. Toward a net neutrality debate that conforms to the 2010s. IEEE Communications Magazine, 54(3):94-99, 2016.

5. P. Maillé and B. Tuffin. Users facing volume-based and flat-rate-based charging schemes at the same time. In IEEE Explore, editor, 8th Latin American Network Operations and Management Symposium (LANOMS 2015), pages 23-26, Joao Pessoa, Brazil, October 2015.

6. H. Pang, L. Gao, Q. Ding, and L. Sun. When data sponsoring meets edge caching: A gametheoretic analysis. CoRR, abs/1709.00273, 2017.

7. R. Somogyi. The economics of zero-rating and net neutrality. CORE Discussion Papers 2016047, Université catholique de Louvain, Center for Operations Research and Econometrics (CORE), 2016.

8. Z. Xiong, S. Feng, D. Niyato, P. Wang, and Y. Zhang. Competition and Cooperation Analysis for Data Sponsored Market: A Network Effects Model. ArXiv e-prints, November 2017.

9. L. Zhang, W. Wu, and D. Wang. Sponsored data plan: A two-class service model in wireless data networks. SIGMETRICS Perform. Eval. Rev., 43(1):85-96, June 2015. 